\title{
The Correlation of Serum Total Testosterone, Sex Hormone Binding Globulin and Free Androgen Index with Athens Insomnia Scale Score in Polycystic Ovary Syndrome
}

\author{
Hartanto Bayuaji ${ }^{1, *}$, Heda Melinda Nazaruddin Nataprawira ${ }^{2}$, Herri Suhari Sastramihardja ${ }^{3}$, \\ Wiryawan Permadi ${ }^{1}$ \\ 'Department of Obstetrics \& Gynecology, Faculty of Medicine, Padjadjaran University, Jl. Eijkman No. 38, Bandung, Indonesia \\ ${ }^{2}$ Department of Child Health, Faculty of Medicine, Padjadjaran University, Jl. Eijkman No. 38, Bandung, Indonesia \\ ${ }^{3}$ Department of Clinical Pharmacology and Therapy, Faculty of Medicine, Padjadjaran University, Jl. Eijkman No. 38, Bandung, Indonesia \\ *Corresponding author. E-mail: tantobayuaji@icloud.com
}

Received date: Apr 12, 2017; Revised: Aug 9, 2017; Accepted: Aug 14, 2017

\section{Abstract}

B

ACKGROUND: Polycystic ovary syndrome (PCOS), a common reproductive endocrinologic disorder in woman, was considered to be related to sleep disturbance. This study is aimed to analyze the correlation between excess androgen markers and Athens Insomnia Scale (AIS) in PCOS.

METHODS: This observational, cross-sectional study of PCOS was conducted to 31 subjects to evaluate the correlation between serum total testosterone, sex hormone binding globulin (SHBG) and free androgen index with the incidence of sleep disturbance using AIS. Sleep disturbance was present if the score $\geq 6$. The correlation between excess androgen markers and sleep disturbance was analyzed using Pearson's coefficient of correlation or Spearman's rho test. Correlation coefficient more than 0.5 with $p<0.05$ was considered significant.
RESULTS: Out of 31 PCOS subjects aged 20-40 years, $39 \%$ subjects had AIS score $\geq 6$. Mean serum testosterone in AIS score $>6$ group was higher than AIS score $<6$ group but not statistically significant (46.68 vs. $28.49 \mathrm{ng} / \mathrm{mL}$, $p>0.05$ ). No significant correlation was found between serum total testosterone, SHBG and free androgen index with AIS score. After adjusting for AIS score, there was the moderate positive correlation between serum total testosterone level although not statistically significant ( $\mathrm{r}=0.54, p=0.07)$.

CONCLUSION: The serum total testosterone level might influence the occurrence of sleep disturbance in PCOS.

KEYWORDS: PCOS, androgen excess, testosterone, sleep disturbance, Athens Insomnia Scale

Indones Biomed J. 2018; 10(1): 46-50

\section{Introduction}

Polycystic ovary syndrome (PCOS) is a common reproductive endocrinologic disorder which could be found in $4-20 \%$ premenopausal women, depending on the criteria used.(1-3) This condition is characterized by oligo- or anovulation, the manifestation of hyperandrogenism, and polycystic ovarian morphology. The pathophysiology is still unclear, however, insulin resistance and hyperandrogenism may play important role.(3-5) Insulin resistance found in PCOS is related to increased serum testosterone and decreased sex-hormone binding globulin (SHBG) level. This may lead to the occurrence of several clinical manifestations due to androgen excess condition (hirsutism, alopecia, and obesity). Obesity may be found in approximately $60 \%$ PCOS women and subject to the occurrence of sleep disturbance. Furthermore, the stressful condition caused by PCOS such as menstrual disturbance and infertility may aggravate the possibility of sleep disturbance. $(3,6,7)$ 
Several studies revealed the presence of sleep disturbance in PCOS, such as excessive daytime sleepiness and increased obstructive sleep apnea (OSA). The exact mechanism of these conditions are not fully understood, but alterations in body fat composition may be the possible cause. Furthermore, sleep disturbance may decrease life quality and probably cause future metabolic disorders.(7-10) To assess sleep disturbance, several tools have been developed. Soldatos, et al., have created a brief, eight question instruments named Athens Insomnia Scale (AIS) based on the International Statistical Classification of Disease and Related Health Problem 10th revision (ICD-10) criteria for sleep disorders. This instrument has been validated and widely used as a tool to detect the presence of sleep disturbance in wide populations. $(7,11,12)$

Although some studies have found the correlation between PCOS and sleep disturbance (7-9,13-15), but data showing the correlation between serum total testosterone, SHBG and free androgen index as markers for androgen excess with AIS score are lacking. This study is aimed to analyze the correlation between those excess androgen markers and the occurrence of sleep disorder in PCOS using AIS.

\section{Methods}

\section{Study Design}

This observational cross-sectional study was conducted to 31 PCOS subjects aged 20-40 years who presented to Assisted Reproductive Unit/Aster Fertility Clinic, Hasan Sadikin Hospital Bandung. The local ethics committee has approved the study protocol, with ethical approval LB.04.01/ A05/EC/110/IV/2017 given from The Ethics Committee for Health Research Hasan Sadikin Hospital.

\section{Inclusion and Exclusion Criteria}

The PCOS was diagnosed using Rotterdam Consensus on Diagnosis Criteria for PCOS. The diagnosis of PCOS was when at least two of these three following conditions were found: 1) oligo- and/or anovulation; 2) clinical and/or biochemical signs of hyperandrogenism; and 3) polycystic ovaries and exclusion of other etiologies, such as congenital adrenal hyperplasia, androgen-secreting tumors or Cushing's syndrome.(16) Subjects who smoke, alcoholics, drink coffee more than $400 \mathrm{~mL}$, were excluded from the study, as well as subjects who used sedative, metformin, corticosteroid, and hormonal contraceptive.

\section{Subjects' Measurement}

All subjects received detailed study information and had given consent before participating in this study. Anthropometric measurement and body mass index calculation were conducted, followed by filling the AIS questionnaire by all subjects. Ten milliliters of peripheral vein blood was collected from all subjects for total serum testosterone, SHBG, and free androgen index assay. Normal values for those excess androgen markers in premenopausal women are: serum total testosterone 8.40-48.1 ng/dL, SHBG 26.1-110 nmol/L and free-androgen index 0.51-6.53\%.(17)

The AIS is a brief questionnaire consists of 8 question to assess the presence of sleep disturbance. Each question has value range $0-3$, with 0 interpreted as no disturbance and 3 as severe problem. Subjects were asked to answer questions in AIS related to sleep disturbance that perceived at least 3 times in a week in the last month. The total sum of the score was interpreted as follow: score less than 6 indicated there was no sleep disturbance, and score more than or equal to 6 indicated the presence of sleep disturbance.(11,12)

\section{Statistical Analysis}

Data were analyzed using Statistical Package for Social Science (SPSS, IBM Co.) version 24. Quantitative data were presented in mean \pm standard deviation, while qualitative data were presented in amount and percentage. To analyze the correlation between total serum testosterone, SHBG and free androgen index with AIS score, Pearson's correlation coefficient test was conducted if data was normally distributed. If the data were not normally distributed or data transformation was not successful, nonparametric Spearman's rho test was used. The correlation coefficient $>0.5$ with $p$ value $<0.05$ was considered significant.

\section{Results}

The characteristics of subjects were listed in Table 1. All subjects were infertile and $61 \%$ were oligomenorrheic. The body mass index mean was $31.21 \mathrm{~kg} / \mathrm{m}^{2}$, with $55 \%$ subjects had body mass index (BMI) more than $30 \mathrm{~kg} / \mathrm{m}^{2}$. Serum total testosterone, SHBG, and free-androgen index level were still in normal range. AIS score median was 4 and $61 \%$ of subjects had AIS score $>6$, which indicated the presence of sleep disturbance.

Meanwhile the correlation analysis of variables with AIS score were shown in Table 2. From Table 2, no significant correlations were found between age, BMI, SHBG level and free-androgen index with AIS score. However, there was 
Table 1. Characteristics of study subjects.

\begin{tabular}{|c|c|}
\hline Characteristics & Results* \\
\hline Age (years) & $30.19 \pm 3.67$ \\
\hline Body weight (kg) & $75.99 \pm 15.19$ \\
\hline Height (cm) & $156.10 \pm 4.74$ \\
\hline BMI $\left(\mathrm{kg} / \mathrm{m}^{2}\right)$ & $31.21 \pm 6.17$ \\
\hline $18.5-24.9$ & $4(13)$ \\
\hline $25-29.9$ & $10(32)$ \\
\hline$\geq 30$ & $17(55)$ \\
\hline
\end{tabular}

\begin{tabular}{ll}
\hline Menstrual cycle (days) & \\
$24-38$ & $12(39)$ \\
$>38$ & $19(61)$ \\
\hline
\end{tabular}

\section{Type of infertility}

Primary

$30(97)$

Secondary

$1(3)$

\begin{tabular}{lc}
\hline Serum total testosterone $(\mathbf{n g} / \mathbf{m L})$ & $26.53(4.80-159.00)$ \\
\hline SHBG $(\mathbf{n m o l} / \mathbf{L})$ & $18.67(6.37-102.70)$ \\
\hline Free-androgen index (\%) & $4.32(0.42-22.18)$ \\
\hline AIS score median & $4(0-14)$ \\
Subjects with score $<6$ & $19(61)$ \\
Subjects with score $\geq 6$ & $12(39)$
\end{tabular}

*Numeric variables with normal distributions were presented in mean \pm standard deviation. Numeric variables with non-normal distributions were presented in median (minimum-maximum). Categorical variables were presented in $\mathrm{n}(\%)$.

Table 2. Correlation analysis of study variables with AIS score.

\begin{tabular}{ll}
\multicolumn{1}{c}{ Variables } & \multicolumn{1}{c}{ AIS score* } \\
\hline Age (years) & $\mathrm{r}=-0.182$ \\
& $p=0.328$ \\
& $\mathrm{n}=31$ \\
\hline BMI $\left(\mathrm{kg} / \mathrm{m}^{2}\right)$ & $\mathrm{r}=0.099$ \\
& $p=0.597$ \\
& $\mathrm{n}=31$ \\
\hline Serum total testosterone $(\mathrm{ng} / \mathrm{mL})$ & $\mathrm{r}=0.326$ \\
& $p=0.074$ \\
& $\mathrm{n}=31$ \\
\hline SHBG (nmol/L) & $\mathrm{r}=0.198$ \\
& $p=0.285$ \\
& $\mathrm{n}=31$ \\
\hline Free-androgen index & $\mathrm{r}=0.166$ \\
& $p=0.373$ \\
& $\mathrm{n}=31$ \\
\hline
\end{tabular}

* Pearson correlation coefficient test

Table 3. The correlation between serum total testosterone level and AIS score.

\begin{tabular}{cccc}
\hline $\begin{array}{c}\text { AIS score } \\
\text { group }\end{array}$ & $\begin{array}{c}\text { Serum total } \\
\text { testosterone level } \\
\text { mean }(\mathbf{n g} / \mathbf{m L})\end{array}$ & $\begin{array}{c}\text { Correlation } \\
\text { coefficient* }\end{array}$ & $\boldsymbol{p}$ value \\
\hline 66 & 46.68 & $\mathrm{r}=0.54$ & 0.07 \\
$<6$ & 28.49 & $\mathrm{r}=-0.4$ & 0.09 \\
\hline * Spearman's rho test & &
\end{tabular}

*Spearman's rho test a weak correlation between serum total testosterone level with AIS score although not reached significance $(\mathrm{r}=0.326$; $p>0.05$ ).

After adjusting the AIS score, analysis of the correlation between serum total testosterone level and AIS score were presented in Table 3. In AIS score $>6$ group, the moderate correlation was found although not reached significance level $(\mathrm{r}=0.54 ; p>0.05)$.

\section{Discussion}

PCOS characterized clinically by the presence of oligo- or anovulation, infertility, hyperandrogenic manifestation, and metabolic disorders such as obesity. Anovulation is $80 \%$ caused by PCOS, making it the leading cause of women infertility and may clinically suspected by the presence of menstrual disorders.(16) Menstrual irregularity is a hallmark of anovulation $(18,19)$, and this was found in this study. All subjects were infertile and $61.29 \%$ complained about oligomenorrhea, which in turn, which reflects anovulation.

Hyperandrogenism is considered an important aspect in PCOS' pathophysiology. This condition might be detected using clinical examination or using laboratory evaluation of excess androgen markers. The common methods to detect hyperandrogenism is to evaluate serum free testosterone and SHBG level, and calculation of free androgen index. (20-22) Hyperandrogenism could manifest as high serum testosterone level or biological activity, low SHBG resulting increased level of free sex steroid hormones, and elevated free androgen index. According to Rosner, et al., free androgen index is a unitless quotient of serum testosterone level divided by serum SHBG level, and it can be served as a surrogate marker to evaluate free testosterone biologic activity. It was a simple and good correlation with physical separation measures in women.(20) Furthermore, the role of serum testosterone in sleep disturbances has been analyzed in several studies. Androgenic receptors were abundantly present in the suprachiasmatic nucleus ( $\mathrm{SCN}$ ), suggesting the role of testosterone in modulating sleep physiology. $(23,24)$

The mean serum total testosterone, SHBG and free androgen index level were in normal range in this study. Elevated circulating androgens were present in $60-80 \%$ of women with PCOS.(22) Hence, androgen excess markers only may not reflect directly the hyperandrogenic condition occurred in PCOS. High BMI and menstrual irregularities, in turn, may represent the effect of hyperandrogenic state found in PCOS. $(3,25,26)$ 
The clinical symptoms caused by PCOS may affect the patient's biopsychosocial life. Women with PCOS have an increased of anxiety and affective disorders, which in turn with coexisting hormonal imbalance may lead to sleep disturbance. Using AIS, there were $39 \%$ subjects whose score $>6$ which indicates the presence of sleep disturbance. Franik, et al., reported there was $12.6 \%$ clinically significant insomnia of their study subjects who complained about sleep disturbance. Furthermore, they also found shorter duration of sleep, and high prevalence of difficulties in falling asleep.They also conclude that screening of sleep disturbance should be a part of medical diagnosis in PCOS.(7)

Sleep disturbance in PCOS has been studied extensively in last decade. Vgontzas and colleagues found 30 times higher risk of developing obstructive OSA in PCOS women.(9) The incidence of OSA in PCOS may increase due to alterations in sex steroids (i.e., high androgen and low estrogen levels) and increased visceral adiposity.(8) Another form of sleep disturbance was excessive daytime sleepiness and insomnia.(7,13) In a larger population consisting 724 PCOS women, sleep disturbance was twice as common in women with PCOS compared with women without PCOS. Furthermore, they also found increasing occurrence of difficulties in falling asleep.(13)

Another possible explanation for high incidence of sleep disorder in PCOS was its relationship with increased abdominal visceral obesity caused by hyperandrogenemia and insulin resistance. Those conditions may alter chest wall and upper airway movement that may lead to a reduction of the functional residual capacity of the lung and increased sleep apnea.(15) Those were in line with subjects characteristics in the present study. The majority of the subjects $(55 \%)$ were obese, which in turn, predispose them to a higher risk of sleep disturbance. On the other hand, other study reported no increase of having OSA in nonobese PCOS women.(27)

No significant correlation exists between subjects' characteristics and excess androgen markers with AIS score in this study. After adjusting the AIS score, subjects in AIS score $>6$ group have a moderate correlation between serum total testosterone level with AIS score. The mean serum total testosterone level in this group was higher than those in AIS score $<6$ group (46.68 vs. $28.49 \mathrm{ng} / \mathrm{mL}, p>0.05$ ). Although not reaching statistical significance, this may reflect the role of testosterone in sleep physiology. In mammals, the central control of circadian rhythm was done by SCN, which is lying above optic chiasma. However, the exact mechanism how SCN controls the sleep-wake cycle and creating sleep architecture are still unclear, but it was believed that SCN regulates circadian timing in physiology and behavior by sending outputs to the another neuroendocrine systems. Androgen receptors were abundantly found in SCN, hence androgenic effects were also important in maintaining circadian timing.(24)

Sleep disturbance now recognized as a determinant factor in women health, especially in the context of menstrual cycle and fertility. Kloss, et al., in their review stated that stress may affect fertility and as well as chronic insomnia to the extent it produces a stress response.(28)

The limitation of this study was the study subjects only consist of PCOS subjects without control from normal, ovulatory women. The limited, small amount sample also may lead to non-significant results in statistical analysis. Since we did not have further data of insulin resistance, the in-depth analysis regarding this condition to sleep disturbance mechanism was not possible. The research regarding the circadian rhythm that might be affected by the androgen excess condition in PCOS warrants further exploration.

Data regarding sleep pattern throughout menstrual cycle is very limited. In the healthy, ovulating women, the sleep homestasis and quality remains stable at different menstrual phases. However, there were some changes in sleep pattern that may subject for further evaluation. (29) Since PCOS was mainly correlated with anovulation, this condition might be explored further. Thus, several aspects i.e., interaction of androgens with other hormones especially melatonin who plays important role in sleep and circadian rhythm in PCOS women also might be analyzed.

\section{Conclusion}

In general, detection and treatment of sleep disturbance in infertile population (especially PCOS), should be an integral part of the management. Clinicians who manage PCOS should become well-informed that besides the established clinical feature, there is another side of management that should be carefully explored in order to provide comprehensive management of PCOS. This indeed may affect biopsychosocial aspects of PCOS management. Furthermore, serum total testosterone level might influence the occurrence of sleep disturbance in PCOS. 


\section{References}

1. Azziz R, Woods KS, Reyna R, Key TJ, Knochenhauer ES, Yildiz BO. The prevalence and features of the polycystic ovary syndrome in an unselected population. J Clin Endocrinol Metab. 2004; 89: 2745-9.

2. Moran L, Teede H. Metabolic features of the reproductive phenotypes of polycystic ovary syndrome. Hum Reprod Update. 2009; 15: 47788.

3. Conway G, Dewailly D, Diamanti-Kandarakis E, Escobar-Morreale HF, Franks S, Gambineri A, et al. The polycystic ovary syndrome: a position statement from the European Society of Endocrinology. Eur J Endocrinol. 2014; 171: P1-29.

4. Lizneva D, Suturina L, Walker W, Brakta S, Gavrilova-Jordan L, Azziz R. Criteria, prevalence, and phenotypes of polycystic ovary syndrome. Fertil Steril. 2016; 106: 6-15.

5. Dewailly D, Lujan ME, Carmina E, Cedars MI, Laven J, Norman RJ, et al. Definition and significance of polycystic ovarian morphology: a task force report from the Androgen Excess and Polycystic Ovary Syndrome Society. Hum Reprod Update. 2014; 20:334-52.

6. Azziz R, Carmina E, Dewailly D, Diamanti-Kandarakis E, EscobarMorreale HF, Futterweit W, et al. The Androgen Excess and PCOS Society criteria for the polycystic ovary syndrome: the complete task force report. Fertil Steril. 2009; 91: 456-88.

7. Franik G, Krysta K, Madej P, Gimlewicz-Pieta B, Oslizlo B, Trukawka J, et al. Sleep disturbances in women with polycystic ovary syndrome. Gynecol Endocrinol. 2016; 32: 1014-7.

8. Tasali E, Van Cauter E, Ehrmann DA. Polycystic ovary syndrome and obstructive sleep apnea. Sleep Med Clin. 2008; 3: 37-46.

9. Vgontzas AN, Legro RS, Bixler EO, Grayev A, Kales A, Chrousos GP. Polycystic ovary syndrome is associated with obstructive sleep apnea and daytime sleepiness: role of insulin resistance. J Clin Endocrinol Metab. 2001; 86: 517-20.

10. Shreeve N, Cagampang F, Sadek K, Tolhurst M, Houldey A, Hill CM, et al. Poor sleep in PCOS; is melatonin the culprit? Hum Reprod. 2013; 28: 1348-53.

11. Soldatos CR, Dikeos DG, Paparrigopoulos TJ. Athens Insomnia Scale: validation of an instrument based on ICD-10 criteria. J Psychosom Res. 2000; 48: 555-60.

12. Soldatos CR, Dikeos DG, Paparrigopoulos TJ. The diagnostic validity of the Athens Insomnia Scale. J Psychosom Res. 2003; 55: 263-7.

13. Moran LJ, March WA, Whitrow MJ, Giles LC, Davies MJ, Moore VM. Sleep disturbances in a community-based sample of women with polycystic ovary syndrome. Hum Reprod. 2015; 30: 466-72.

14. Ehrmann DA. Metabolic dysfunction in PCOS: Relationship to obstructive sleep apnea. Steroids. 2012; 77: 290-4.

15. El-Sharkawy AA, Abdelmotaleb GS, Aly MK, Kabel AM. Effect of metformin on sleep disorders in adolescent girls with polycystic ovarian syndrome. J Pediatr Adolesc Gynecol. 2014; 27: 347-52.
16. Rotterdam, ESHRE-ASRM-Sponsored PCOS consensus workshop group. Revised 2003 consensus on diagnostic criteria and long-term health risks related to polycystic ovary syndrome (PCOS). Hum Reprod. 2004; 19: 41-7.

17. Vermeulen A, Verdonck L, Kaufman JM. A critical evaluation of simple methods for the estimation of free testosterone in serum. J Clin Endocrinol Metab. 1999; 84: 3666-72.

18. Munro MG, Critchley HO, Fraser IS. The FIGO systems for nomenclature and classification of causes of abnormal uterine bleeding in the reproductive years: who needs them? Am J Obstet Gynecol. 2012; 207: 259-65.

19. Practice Committee of the American Society for Reproductive Medicine. Diagnostic evaluation of the infertile female: a committee opinion. Fertil Steril. 2015; 103: e44-50.

20. Rosner W, Auchus RJ, Azziz R, Sluss PM, Raff H. Position statement: Utility, limitations, and pitfalls in measuring testosterone: an Endocrine Society position statement. J Clin Endocrinol Metab. 2007; 92: 405-13.

21. Tziomalos K, Katsikis I, Papadakis E, Kandaraki EA, Macut D, Panidis D. Comparison of markers of insulin resistance and circulating androgens between women with polycystic ovary syndrome and women with metabolic syndrome. Hum Reprod. 2013; 28: 785-93.

22. Azziz R, Carmina E, Dewailly D, Diamanti-Kandarakis E, EscobarMorreale HF, Futterweit W, et al. Positions statement: criteria for defining polycystic ovary syndrome as a predominantly hyperandrogenic syndrome: an Androgen Excess Society guideline. J Clin Endocrinol Metab. 2006; 91: 4237-45.

23. Karatsoreos IN, Silver R. Minireview: The neuroendocrinology of the suprachiasmatic nucleus as a conductor of body time in mammals. Endocrinology. 2007; 148: 5640-7.

24. Mong JA, Baker FC, Mahoney MM, Paul KN, Schwartz MD, Semba $\mathrm{K}$, et al. Sleep, rhythms, and the endocrine brain: influence of sex and gonadal hormones. J Neurosci. 2011; 31: 16107-16.

25. Rosenfield RL, Ehrmann DA. The pathogenesis of polycystic ovary syndrome (PCOS): the hypothesis of PCOS as functional ovarian hyperandrogenism revisited. Endocr Rev. 2016; 37: 467520 .

26. Legro RS. Obesity and PCOS: implications for diagnosis and treatment. Semin Reprod Med. 2012; 30: 496-506.

27. Mokhlesi B, Scoccia B, Mazzone T, Sam S. Risk of obstructive sleep apnea in obese and nonobese women with polycystic ovary syndrome and healthy reproductively normal women. Fertil Steril. 2012; 97: 786-91.

28. Kloss JD, Perlis ML, Zamzow JA, Culnan EJ, Gracia CR. Sleep, sleep disturbance, and fertility in women. Sleep Med Rev. 2015; 22: 78-87.

29. Shechter A, Boivin DB. Sleep, hormones, and circadian rhythms throughout the menstrual cycle in healthy women and women with premenstrual dysphoric disorder. Int J Endocrinol. 2010; 2010: 259345. doi: 10.1155/2010/259345. 